\title{
Transferência Tecnológica na Pecuária Leiteira: um estudo sob o enfoque dos produtores das Regiões do Vale do Mucuri (MG) e do Extremo Sul da Bahia
}

\author{
Technological Transfer in Dairy Cattle: a study under the approach \\ of the producers of the Regions of the Mucuri Valley (MG) and the \\ Extreme South of Bahia
}

\author{
Laíse Nunes Maciel ${ }^{1}$ \\ Lucas Xavier Trindade ${ }^{2}$ \\ Fárlei Cosme Gomes dos Santos ${ }^{3}$ \\ João Pedro de Castro Nunes Pereira ${ }^{1}$ \\ ${ }^{1}$ Universidade Estadual de Santa Cruz, BA, Brasil \\ ${ }^{2}$ Universidade de São Paulo, Programa de Pós-graduação em Administração, SP, Brasil \\ ${ }^{3}$ Centro Estadual de Educação Profissional do Chocolate Nelson Schaun, Ilhéus, BA, Brasil
}

\begin{abstract}
Resumo
Embora a tecnologia desempenhe papel de destaque para a competitividade da agropecuária brasileira, existem segmentos e regiões situados à margem do desenvolvimento em função da baixa produtividade e da pobreza em decorrência do reduzido aporte tecnológico existente nas unidades produtivas rurais. Esta investigação do tipo survey aplicado a 20 produtores teve como objetivo diagnosticar a percepção desses agentes frente às principais condicionantes associadas ao fenômeno da transferência de tecnologia para unidades de produção de leite das localidades do Vale do Mucuri (MG) e do Extremo Sul da Bahia. Ficou evidenciado que, embora os produtores tenham disposição favorável para adotarem tecnologias e a considerem um relevante fator de produção para a pecuária leiteira, outras condicionantes não se mostram favoráveis à concretização da transferência de tecnologia $e$ denotam fraquezas existentes nesse processo. Foi constatado também que inexistem diferenças significativas de percepção dos produtores frente às condicionantes em função da localidade e do tamanho de suas respectivas unidades de produção.
\end{abstract}

Palavras-chave: Tecnologia. Transferência. Pecuária.

\begin{abstract}
Although technology plays a prominent role in the competitiveness of Brazilian agriculture, there are segments and regions located on the fringes of development due to low productivity and poverty due to the low technological support existing in rural production units. This survey research applied to 20 producers aimed to diagnose the perception of these agents regarding the main conditions associated with the phenomenon of technology transfer to milk production units in the towns of Vale do Mucuri (MG) and the far south of Bahia. It is evidenced that although producers have a favorable disposition to adopt technologies and consider it a relevant production factor for dairy farming, other conditions are not favorable to the achievement of technology transfer and denote weaknesses existing in this process. It was also found that there are no significant differences in the perception of producers regarding the conditions depending on the location and size of their respective production units.
\end{abstract}

Keywords: Technology. Transfer. Dairy Cattle

Área Tecnológica: Transferência de Tecnologia. 


\section{Introdução}

A partir da década de 1950, o processo de modernização da agricultura passa a ser significativamente impulsionado com vistas a aumentar a sua produtividade por meio da incorporação de novas tecnologias. As instituições de ciência e tecnologia (ICTs) possuem papel fundamental na produção e transmissão de conhecimento, a fim de desenvolver novas tecnologias e aprimorar as já existentes (BARBOSA et al., 2019), representando papel importante para o desenvolvimento da economia devido a sua capacidade de geração de conhecimentos e de tecnologias (FERNANDES et al., 2018). No contexto da modernização agrícola nacional, a participação das ICTs tem grande destaque, segundo Matos e Pessoa (2011), a modernização se tornou viável devido aos incentivos promovidos pelo Estado por meio de investimentos nas ciências agrárias, órgãos de apoio e suporte à produção, programas de assistência e extensão $e$ oferta de crédito agrícola. Um dos órgãos mais significativos criados nesse período foi a Empresa Brasileira de Pesquisa Agropecuária (EMBRAPA), voltada ao desenvolvimento de tecnologias e de dados técnico-científicos para a agricultura e a pecuária brasileira (GASQUES; VIEIRA FILHO; NAVARRO, 2010).

De acordo com Lisita (2005), o Brasil, no ano de 1960, assumiu um padrão produtivo agropecuário instituído devido a união entre o ensino (as universidades e escolas técnicas), a pesquisa (universidades e centros de pesquisa), e a extensão rural (agentes de governo e setor privado). Essa união teve como produto a inserção dos pacotes tecnológicos voltados para o aproveitamento intensivo de máquinas e insumos com o intuito de obter ganhos de produtividade.

A extensão rural, especialmente quando desenvolvida por empresas públicas, tem como principal objetivo provocar e fortalecer a distribuição de tecnologias produzidas pela pesquisa agropecuária. Já quando desenvolvida por empresas privadas, normalmente visa a apresentar novas tecnologias associadas a novos produtos - direta ou indiretamente - e a garantir que os produtores sejam capazes de absorvê-las e aplicá-las em suas atividades produtivas cotidianas. Para Dereti (2009), a transferência se concretiza quando os receptores da tecnologia estão são capazes de absorver transformar, adequar e ampliá-la conforme a sua utilidade. Sob essa abordagem, considera-se como sendo o principal foco do processo de transferência os agentes de produção responsáveis pelo processo de incorporação das tecnologias nos processos produtivos.

No entanto, apesar de o Brasil se destacar mundialmente pela exportação de produtos agrícolas associados a altos padrões tecnológicos de produção, ainda existem considerável quantidade de regiões que não conseguiram incorporar os padrões tecnológicos em suas atividades. A heterogeneidade na agropecuária ainda persiste devido às diferenças na adoção e na difusão da tecnologia, do acesso à terra, da infraestrutura, das políticas públicas e do acesso ao crédito (FORNAZIER; VIEIRA FILHO, 2012).

Esse cenário pode ser constatado no segmento da pecuária leiteira, no qual existe considerável heterogeneidade entre as unidades rurais de produção. Segundo Carvalho et al. (2009), unidades de produção de subsistência com pouca tecnologia embarcada convivem com unidades produtivas de elevado aporte tecnológico. No Extremo Sul da Bahia, a produção de leite passa a representar a partir de 1989 uma alternativa para a produção de cacau devido à crise instaurada na lavoura provocada por fatores agronômicos e econômicos (COSTA, 2012). Já na região do Vale do Mucuri (MG), a produção leiteira representa uma das principais atividades econômicas. 
Segundo Vieira Filho (2014), o maior desafio para a trajetória tecnológica da agropecuária está vinculado à disseminação e à assimilação desse componente produtivo pelos produtores situados à margem do desenvolvimento. Sendo assim, a transferência de tecnologia agropecuária se constitui em importante instrumento de cooperação, o que poderá se configurar como uma das possibilidades para que ocorra de forma efetiva a contribuição para promoção da competitividade e do desenvolvimento do setor.

Nesse contexto, os resultados divulgados neste trabalho pretendem contribuir para o diagnóstico e a compreensão da dinâmica envolvida no processo de transferência tecnológica na agropecuária leiteira sob o enfoque dos produtores. Desse modo, busca-se compreender como esses agentes percebem e avaliam os aspectos relacionados à transferência de tecnologia. No ambiente de estudo, os produtores se constituem em agentes que desempenham papel crítico por representarem os tomadores de decisão sobre as tecnologias absorvidas nas propriedades rurais. Portanto, a investigação se constitui em diagnóstico da percepção dos produtores às condicionantes de transferência de tecnologia agropecuária.

\section{Transferência de Tecnologia no Ambiente Agropecuário}

Para Hayami e Ruttan (1985), a transferência de tecnologia é crucial para o desenvolvimento agrícola. Apenas o desenvolvimento tecnológico não representa condição suficiente, fazendo-se necessário incluir o processo de transporte da tecnologia (OLIVEIRA; SEGATTO, 2009). A transferência tecnológica se constitui, portanto, em intercâmbio ou transmissão de conhecimentos técnicos entre dois ou mais agentes, pressupondo a existência de um controlador da tecnologia (concedente) e, de outro, um dependente (receptor) que necessita da tecnologia (ASSAFIM, 2010). Para Miranda e Simeão (2004), na maioria dos casos, o que é transferido é a competência de reproduzir e de utilizar a tecnologia, dificilmente é transferido o conhecimento que originou a tecnologia, que, por sua vez, acaba por gerar dependências tecnológicas, muitas vezes, cruciais ao bom desempenho dos negócios.

No contexto rural, a transferência tecnológica se associa aos serviços de assistência e extensão rural sob maior responsabilidade do setor público, conduzidos, principalmente, aos pequenos e médios produtores. Porém, as cooperativas, as associações, os sindicatos e as indústrias também desenvolvem esse tipo de serviço (MENDES; BUAINAIN, 2015).

Shaw (1987) entende que a transferência de tecnologia seja, em grande parte, uma forma de comunicação sob responsabilidade do serviço de extensão rural. Segundo Díaz e Hernández (2002), o objetivo da transferência de tecnologia agropecuária consiste em propor técnicas que, adotadas pelos produtores rurais, propiciem aumento de produção, aprimoramento da qualidade dos produtos, combinação mais eficiente dos fatores de produção e uso racional dos recursos naturais.

A assistência e a extensão rural representam os mecanismos mais aplicados no processo de transferência tecnológica e de acesso à informação relevante aos agricultores (NOCE, 2017) e, atrelam-se, geralmente, às políticas públicas de promoção do desenvolvimento rural que, por sua vez, dependem da existência e da capacidade de intervenção de profissionais que orientem localmente as mudanças em termos de políticas (DIAS, 2008). 
Uma das modalidades utilizadas para a transferência de tecnologia agropecuária está associada à difusão tecnológica que busca difundir conhecimentos, bens e serviços isentos de proteção, similares a bens públicos, os quais são apropriados pelos produtores rurais a partir dos agentes de extensão e assistência técnica rural, dias de campo, unidades de demonstração, cursos, palestras, publicações e mídias (GOMES; ATRASAS, 2005).

Porém, Vital e Silva Neto (2006) entendem que apenas a demonstração dos procedimentos de uso da tecnologia não indica que a sua utilização e adoção serão efetivas. Complementarmente, para Silva, Kovaleski e Gaia (2013), a transferência não pode ser considerada como o simples repasse de conhecimentos, mas um processo que se inicia com a identificação da tecnologia a partir de uma demanda, com a seleção das ferramentas de transferência de tecnologia ajustadas a cada caso e a adoção pelo público de interesse.

Independentemente da classificação acadêmica que pode ser adotada em função do foco do estudo desenvolvido, alguns fatores, como tamanho da propriedade e disponibilidade dos receptores das tecnologias, proximidade dos centros de desenvolvimento tecnológico, entre outros, são essenciais na delimitação das estratégias de transferência tecnológica (DERETI, 2009; SOUZA FILHO et al., 2011).

\section{Metodologia}

Esta pesquisa se caracteriza como um survey que, segundo Fink (2017), representa um método que recolhe das pessoas de modo direto as informações referentes a um tema proposto e que, segundo Babbie (2001), possibilita a estruturação transparente de um padrão lógico, explicando as razões, as características ou as relações observadas sobre o assunto. O instrumento de pesquisa utilizado foi um questionário estruturado com base no levantamento bibliográfico apresentado anteriormente que aborda as condicionantes de influência identificadas na revisão de literatura apresentadas no Quadro 1.

Quadro 1 - Condicionantes a transferência tecnológica na agropecuária estudadas

\begin{tabular}{|c|c|}
\hline Condicionantes estudadas & Referências Bibliográficas \\
\hline $\begin{array}{l}1 \text { Disposição em adotar novas tecnologias adequadas } \\
\text { ao seu perfil ou necessidades dos pecuaristas. } \\
\mathbf{2} \text { Adequação da linguagem adotada } \\
\text { pelos agentes no processo de TT. } \\
\mathbf{3} \text { Frequência de contatos com os agentes } \\
\text { de desenvolvimento tecnológico. } \\
4 \text { Importância da extensão e assistência técnica } \\
\text { rural para a transferência de tecnologia. }\end{array}$ & $\begin{array}{l}\text { Souza Filho et al. (2011); Mendes } \\
\text { (2015); Possas; Salles-Filho e } \\
\text { Silveira (1994); Noce (2017). }\end{array}$ \\
\hline
\end{tabular}

Fonte: Elaborado pelos autores deste artigo

A amostra foi do tipo não probabilística composta de 20 produtores das localidades estudadas, escolhidos por critérios de conveniência e disponibilidade de participarem da pesquisa e pelo critério do tipo "bola de neve", quando alguns indivíduos participantes indicam novos possíveis participantes para a pesquisa (BICKMAN; ROG, 1997). 
A análise dos resultados foi dividida em duas etapas com o auxílio do software estatístico IBM SPSS Satistics 15. A primeira etapa, descritiva, apresenta a distribuição percentual das percepções dos produtores para cada uma das condicionantes estudadas. Em etapa seguinte foi realizado um teste de Kruskal-Wallis com a finalidade de identificar possíveis diferenças significativas de percepção dos produtores às condicionantes estudas em função da localidade e do tamanho das unidades de produção.

\section{Resultados e Discussões}

Perfil da Amostra - os pecuaristas que compõem a amostra são predominantemente do município baiano de Itanhém (50\%) na Bahia, os demais produtores da amostra pertencem a municípios de Minas Gerais, portanto, metade da amostra foi coletada na Bahia e a outra metade em Minas Gerais, conforme mostra a Figura 1.

Figura 1 - Número de Entrevistados por Município

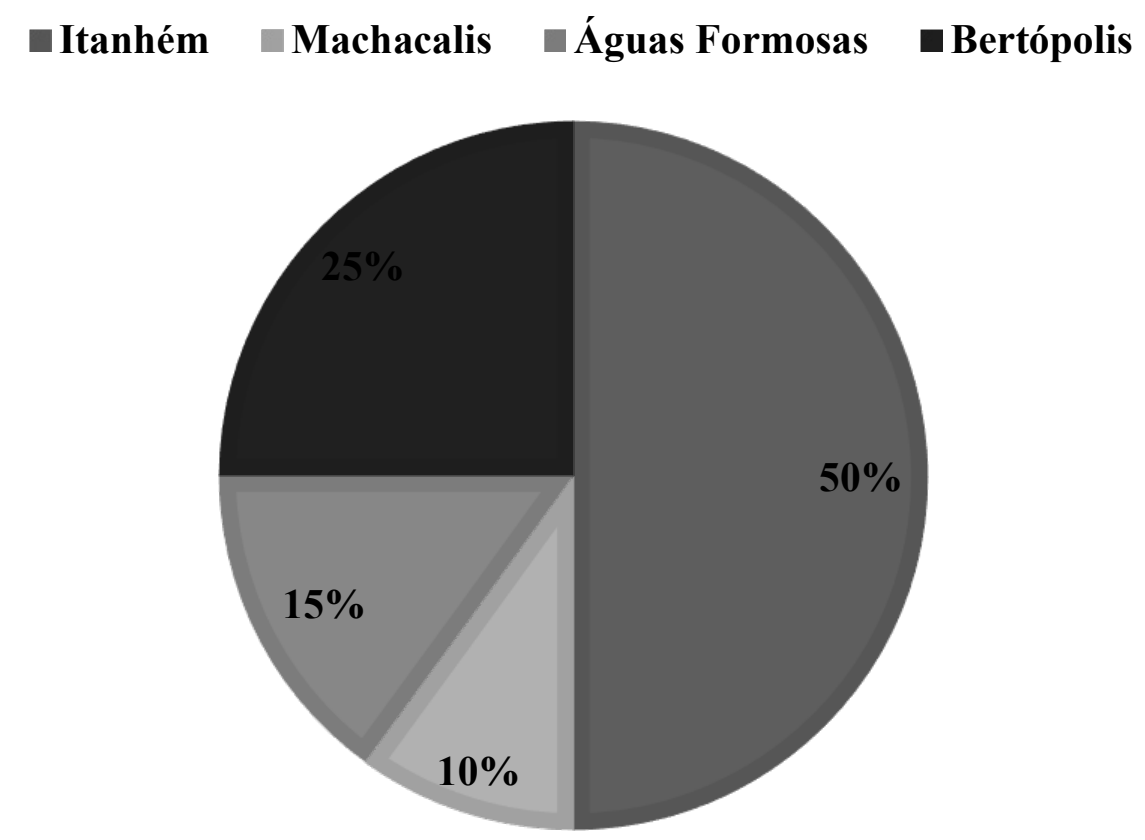

Fonte: Elaborada pelos autores deste artigo

A estratificação da amostra estudada revelou a presença de produtores com diferentes tamanhos de propriedade de acordo com a classificação definida na Lei n. 8.629, de 1993 (BRASIL, 1993). Segundo essa classificação, as propriedades rurais definidas em minifúndio, pequena propriedade, média propriedade e grande propriedade, as quais possuem respectivamente área inferior a 1 módulo fiscal, entre 1 a 4 módulos fiscais, superior a 4 módulos e até 15 módulos fiscais, e área superior a 15 módulos fiscais. Essa configuração implica na consideração de estratégias diferenciadas no processo de transferência tecnológica conforme destacado por Dereti (2009) e Souza Filho et al. (2011). A Figura 2 revela que a maioria das propriedades representadas na amostra é composta de produtores de minifúndios (40\%), pequenas propriedades (30\%), médias propriedades $(20 \%)$ e apenas $10 \%$ de grandes produtores. 
Figura 2 - Classificação das Propriedades em Estudo

- Minifúndio $\square$ Pequena Propriedade $\quad$ Média Propriedade $\quad$ Grande Propriedad

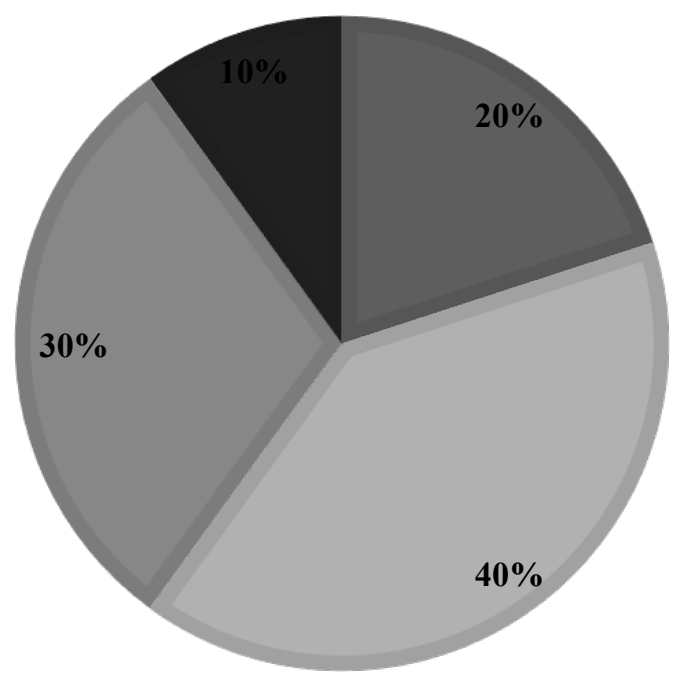

Fonte: Elaborada pelos autores deste artigo

Para Souza Filho et al. (2011), o tamanho da área de produção influência no processo de absorção de tecnologia que, por sua vez, implicará diretamente nas estratégias de transferência tecnológica no ambiente rural. Para esses autores, algumas tecnologias excedem a capacidade de utilização em propriedades menos extensas e, em alguns casos, podem representar um custo elevado para sua adoção e operacionalização, fazendo-as em muitos casos inacessíveis aos pequenos produtores (SOUZA FILHO et al., 2011).

Transferência de tecnologia: percepção dos produtores frente às condicionantes associadas a transferência de tecnologia - com relação às condicionantes associadas à transferência de tecnologia, Noce (2017) destaca que o interesse e a disposição em adotar novas tecnologias são essenciais nesse processo. Os resultados revelados na Figura 3 sugerem um perfil de produtores favoráveis à transferência de tecnologia, a medida em que não existe indicativo de disposição contrária à adoção de novas tecnologias.

Figura 3 - Percepções acerca das condicionantes de transferência tecnológica adotadas neste estudo

\begin{tabular}{|c|c|c|c|}
\hline $\begin{array}{l}\text { Disposição em adotar novas tecnologias adequadas ao seu } \\
\text { perfil ou necessidades dos pecuaristas; }\end{array}$ & \multicolumn{2}{|c|}{$65 \%$} & $35 \%$ \\
\hline $\begin{array}{l}\text { Adequação da linguagem adotada pelos agentes no processo } \\
\text { de TT }\end{array}$ & $35 \%$ & $37,50 \%$ & $27,5 \%$ \\
\hline $\begin{array}{l}\text { Frequência de contatos com os agentes de desenvolvimento } \\
\text { tecnológico }\end{array}$ & $35 \%$ & $45 \%$ & $20 \%$ \\
\hline $\begin{array}{c}\text { Importância da extensão e assistência técnica rural para a } \\
\text { transferência de tecnologia }\end{array}$ & $35 \%$ & $45 \%$ & $20 \%$ \\
\hline
\end{tabular}

Fonte: Elaborada pelos autores deste artigo 
Contudo, os resultados obtidos para os demais fatores contemplados neste estudo revelam um contexto menos propício ao sucesso do processo de transferência tecnológica. Para a condicionante de "Linguagem adotada pelos agentes no processo TT", 35\% dos produtores entrevistados entendem que essa condicionante não esteja adequada aos objetivos de transferência de tecnológica. Para apenas $27,5 \%$ dos produtores, a linguagem utilizada pelos agentes de transferência representa condicionante adequada.

Porém, 37,5\% dos entrevistados não indicam que a linguagem dos agentes seja adequada ou inadequada. Esse resultado pode ser representativo da desconsideração de diferentes estratégias de transferência, incluindo a utilização de linguagens específicas para públicos determinados. A utilização de linguagem adequada ao perfil dos produtores foi discutida por Rogers (2013) que destaca ser esse um fator essencial para o sucesso da transferência tecnológica no ambiente rural.

Com relação à frequência de contatos com os agentes de desenvolvimento tecnológico para a transferência, os dados da Figura 3 indicam que apenas $20 \%$ dos produtores entrevistados atestam a eficácia desses agentes. Isso sugere um distanciamento entre os agentes de extensão e os produtores, o que, em tese, desfavorece a transferência de tecnologia para as unidades produtivas investigadas. Isso fica também evidente devido a $45 \%$ dos produtores indicarem que têm contato apenas "às vezes" com esses agentes, enquanto apenas $20 \%$ dos produtores sugerem ter contatos frequentes com os agentes de desenvolvimento tecnológico. $\mathrm{O}$ resultado para essa condicionante se associa à possibilidade de ser produto do modelo de desintegração existente entre a pesquisa e a extensão agropecuária existente no Brasil (NOCE, 2017).

Os serviços de extensão e de assistência técnica se constituem nos principais mecanismos de transferências de tecnologias e de acesso à informação relevante aos agricultores (SOUZA FILHO, 2011; MENDES, 2015; NOCE, 2017). Para 35\% dos produtores, esses serviços indicam não terem importância, enquanto apenas $20 \%$ dos entrevistados entendem que a extensão $e$ a assistência sejam relevantes para a transferência de tecnologia para suas propriedades. Esse resultado se mostra vinculado à pouca percepção existente entre os produtores de frequência de contatos com os agentes de desenvolvimento tecnológico e ao perfil das propriedades, as quais são caracterizadas como pequenas e dependentes quase que exclusivamente dos serviços públicos.

Relação entre local e tamanho das propriedades rurais e a percepção dos produtores - a verificação de possíveis diferenças de percepção entre os produtores segundo a localidade e o tamanho das unidades de produção foi realizada a partir do teste de Kruskal-Wallis. Os resultados dessas análises são apresentados nos Quadros 2 e 3, considerando-se um nível de significância de $5 \%$. Os resultados apresentados no Quadro 2 consideram a localização (municípios), enquanto o Quadro 3 considera o tamanho das unidades de produção contempladas no estudo.

Quadro 2 - Diferenças de percepção em função da localização das unidades agrícolas de produção

\begin{tabular}{|c|c|c|c|c|}
\hline CondicionANTE & $\begin{array}{c}\text { Disposição EM } \\
\text { ADOTAR NOVAS } \\
\text { TECNOLOGIAS }\end{array}$ & $\begin{array}{c}\text { FREQUÊNCIA DE } \\
\text { AdEQUAÇÃo DA } \\
\text { LINGUAGEM }\end{array}$ & $\begin{array}{c}\text { CONTATOS AGENTES } \\
\text { DE DESENVOLVIMENTO } \\
\text { TECNOLÓGICO }\end{array}$ & $\begin{array}{c}\text { IMPORTÂNCIA DA } \\
\text { EXTENSÃo E } \\
\text { ASSISTÊNCIA RURAL }\end{array}$ \\
\hline Qui-quadrado & 2,035 & 2,494 & 3,930 & 5,637 \\
\hline Nível de Significância & 0,565 & 0,476 & 0,269 & 0,131 \\
\hline
\end{tabular}

Fonte: Elaborado pelos autores deste artigo 
Os dados do Quadro 2 revelam que não foi identificada significância para nenhum dos fatores estudados. Isso indica que a localização das unidades produtivas não influenciou, neste estudo, a percepção e a avaliação das condicionantes associadas à transferência de tecnologia no ambiente estudado. Isso significa que as percepções frente às condicionantes consideradas neste estudo são similares independentemente do município onde estão sediadas as propriedades rurais (unidades de produção) que compuseram o universo estudado.

Quadro 3 - Diferenças de percepção em função do tamanho das unidades agrícolas de produção.

\begin{tabular}{|c|c|c|c|c|}
\hline $\begin{array}{l}\text { Condicionante } \\
\text { Estatística }\end{array}$ & $\begin{array}{c}\text { DISPOSIÇÃO EM } \\
\text { ADOTAR NOVAS } \\
\text { TECNOLOGIAS }\end{array}$ & $\begin{array}{l}\text { ADEQUAÇÃO DA } \\
\text { LINGUAGEM }\end{array}$ & $\begin{array}{l}\text { FREQUÊNCIA DE CON- } \\
\text { TATOS AGENTES DE } \\
\text { DESENVOLVIMENTO } \\
\text { TECNOLÓGICO }\end{array}$ & $\begin{array}{l}\text { IMPORTÂNCIA DA } \\
\text { EXTENSÃO E } \\
\text { ASSISTÊNCIA } \\
\text { RURAL }\end{array}$ \\
\hline Qui-quadrado & 3,128 & 7,207 & 3,948 & 1,775 \\
\hline Nível de Significância & 0,372 & 0,066 & 0,267 & 0,620 \\
\hline
\end{tabular}

Fonte: Elaborado pelos autores deste artigo

Similarmente, o Quadro 3 revela também que não há significância para nenhuma das condicionantes estudadas. Esses resultados demonstram que o tamanho das unidades produtivas não influenciou a percepção dos produtores frente às condicionantes associadas a transferência de tecnologia no ambiente estudado. Esse resultado revela que a percepção e a análise das condicionantes consideradas neste estudo não estiveram associadas ao tamanho das unidades agrícolas de produção, sugerindo que esse determinante, neste estudo, não influenciou o perfil de resposta dos produtores investigados.

O teste de Kruskal-Wallis indica, portanto, que, neste estudo especificamente, os determinantes de localização e de tamanho das unidades de produção não influenciaram a percepção dos produtores frente às condicionantes estudadas. A disposição dos produtores em adotarem novas tecnologias, a adequação da linguagem, a frequência de contatos com os agentes de desenvolvimento tecnológico e a importância da extensão e assistência são similares entre os produtores de leite, tanto do Extremo Sul da Bahia quanto do Vale do Mucuri (MG), e entre os diferentes portes de propriedades rurais.

\section{Considerações Finais}

A investigação, ao avaliar a percepção dos produtores frente às condicionantes de transferência tecnológica para os pecuaristas de leite no Vale do Mucuri (MG) e Extremo Sul da Bahia, constitui-se em diagnóstico e contribui para a discussão teórica desse processo no ambiente agropecuário de produção de leite nessas duas localidades.

Os resultados mostraram que, embora os produtores tenham disposição em adotar tecnologias, outras condicionantes não se mostram favoráveis à concretização da transferência de tecnologia e denotam, portanto, fraquezas existentes nesse processo.

As condicionantes investigadas neste estudo são pontuadas pela bibliografia como essenciais ao processo de transferência tecnológica no ambiente agropecuário. A linguagem utilizada no 
processo, a frequência de contatos com os agentes de transferência e a importância dos serviços de extensão e assistência técnica se mostraram, portanto, serem fragilidades identificadas.

O teste de Kruskal-Wallis indica que, neste estudo especificamente, os determinantes de localização e tamanho das unidades de produção não influenciaram a percepção dos produtores frente às condicionantes estudadas. Portanto, as percepções diante das condicionantes propostas para a avaliação são similares entre os produtores de leite, tanto do Extremo Sul da Bahia quanto do Vale do Mucuri (MG), e entre os diferentes portes de propriedades rurais.

\section{Referências}

ASSAFIM, H. M. de L. A Transferência de Tecnologia no Brasil: aspectos contratuais e concorrenciais da Propriedade Industrial. Rio de Janeiro: Editora Lumen Juris, 2010.

BABBIE, Earl. Métodos de Pesquisas de Survey. Belo Horizonte: Editora UFMG, 2001. 519p.

BARBOSA, A. M. A. et al. Um panorama do desempenho em inovação no Brasil e a busca por boas práticas de gestão na transferência de tecnologia (TT) nas instituições de ciência e tecnologia (ICT) do Brasil. Cadernos de Prospecção, Salvador, v. 12, n. 3, p. 504-522, set. 2019.

BICKMAN, L. B.; ROG, D. J. Handbook of applied research methods. Thousand Oaks: Sage, 1997. 580p.

BRASIL. Lei n. 8629, de 25 de fevereiro de 1993. Dispõe sobre a regulamentação dos dispositivos constitucionais relativos à reforma agrária, previstos no Capítulo III, Título VII, da Constituição Federal. Diário Oficial da União, Brasília, DF, 25 fev. 1993.

BURGELMAN, R. A.; CHRISTENSEN, C. M.; WHELLWRIGHT, S. C. Gestão estratégica da tecnologia e da inovação: conceitos e soluções. 5. ed. [S.l.]: Mcgraw Hill, 2012.

CAPORAL, F. R.; FIALHO, J. R. D. A extensão rural e os limites à prática dos extensionistas do serviço público. Extensão rural, Santa Maria, n. 1, 1993.

CARVAlHO, G. R. et al. Competitividade da cadeia produtiva do leite em pernambuco. 1. ed. Juiz de Fora: Embrapa, 2009.

COSTA, F. M. Políticas públicas e atores sociais na evolução da cacauicultura baiana. 2013. 213f. Tese (Doutorado) - Universidade Federal Rural do Rio de Janeiro, Instituto de Ciências Humanas e Sociais, Rio de Janeiro, 2012.

DAMANPOUR, F. Organizational innovation: the meta-analysis of effects of determinants and moderators. Academy Management Journal, [S.I.], v. 34, n. 3, p. 555-590, 1991.

DERETI, R. M. Transferência e validação de tecnologias agropecuárias a partir de instituições de pesquisa. Revista Desenvolvimento e Meio Ambiente, Paraná, n. 19, p. 29-40, jan.-jun., 2009.

DIAS, M. M. Políticas públicas de extensão rural e inovações conceituais: limites e potencialidades. Perspectivas em Políticas Públicas, Belo Horizonte, v. 1, n. 1, p. 101-114, jan.-jun., 2008.

DÍAZ, E. C.; HERNÁNDEZ, A. V. Una metodología para evaluar el proceso de generación, de transparencia y adopción de tecnologia. Agrociencia, Chapingo, v. 36, n. 1, p. 123-130, 2002. 
FERNANDES, R. F. et al. Práticas de transferência de tecnologia: uma análise multicasos. Cadernos de Prospecção, Salvador, v. 11, n. 5, Edição Especial, p. 1.342-1.359, dez., 2018.

FINK, A. How to conduct surveys: a step by step guide. 6. ed. California: Sage, 2017.

FORNAZIER, A.; VIEIRA FILHO, J. E. R. Heterogeneidade estrutural no setor agropecuácio brasileiro: evidências a partir do censo agropecuário de 2006. Brasília: IPEA, n. 1.708, 2012. (Texto para Discussão, IPEA)

GASQUES, J.; VIEIRA FILHO, J.; NAVARRO, Z. A agricultura brasileira: desempenho, desafios e perspectivas. Brasília: IPEA/MAPA, 2010. p. 298.

GOMES, G. C.; ATRASAS, A. L. Diretrizes para transferência de tecnologia: modelo de incubação de empresas. Série Documentos n. 2. Brasília: EMBRAPA Informação Tecnológica, 2005.

HAYAMI, Y.; RUTTAN, V. W. Agricultural development: an international perspective. rev. and expand. ed. Baltimore: The J. Hopkins University Press, 1985.

LISITA, F. O. Considerações sobre a extensão rural no Brasil. EMBRAPA Pantanal, Corumbá, MS, n. 77, p. 1-3, abril de 2005.

MATOS, P. F.; PESSOA, V. L. S. A modernização da agricultura no brasil e os novos usos do território. Geo UERJ, Rio de Janeiro, v. 2, n. 22, p. 290-322, 2011.

MENDES, C. I. C. Transferência de Tecnologia da Embrapa: rumo à inovação. 2015. 415p. Tese (Doutorado) - Universidade Estadual de Campinas, Instituto de Economia, Campinas, 2015.

MENDES, C. I. C.; BUAINAIN, A. M. Transferência de tecnologia: análise além das fronteiras da Embrapa. In: CONGRESSO LATINO-IBEROAMERICANO DE GESTÃO DA TECNOLOGIA, 16. 2015, Porto Alegre. Anais [...]. Porto Alegre: Universidade Federal do Rio Grande do Sul, 2015.

MIRANDA, A.; SIMEÃO, E. Transferência de informação e transferência de tecnologia no modelo de comunicação extensiva: a babel.com. Inf. Cult. Soc., Ciudad Autónoma de Buenos Aires, n. 10, p. 27-40, jan.-jun., 2004.

NOCE, M. A. Análise do processo de transferência de tecnologia no sistema de integração lavoura-pecuária-floresta, para agricultores familiares na região central de Minas Gerais. 2017. 162f. Tese (Doutorado) - Departamento de Economia Rural, Universidade Federal de Viçosa, Viçosa, 2017.

OLIVEIRA, S. A; SEGATTO, A. P. Transferência de tecnologia e conhecimento sob a lente estruturacionista: uma integração temática. RAE Eletrônica, [S.l.], v. 8, n. 2, p. 1-27, 2009.

POSSAS, M.; SALLES-FILHO, Sergio; SILVEIRA, José Maria da. An evolutionary approach to technological innovation in agriculture: some preliminary remarks. Cadernos de Ciência \& Tecnologia, [S.l.], v. 11, n. 1-3, p. 9-31, 1994.

ROGERS, E. M. Diffusion of innovations. 5. ed. The Free Press, Nova Iorque, 2013.

SHAW, A. B. Approaches to agricultural technology adoption and consequences of adoption in the third world: a critical review. Geoforum, [S.l.], v. 18, n. 1, p. 1-19, 1987.

SILVA, L. C. S.; KOVALESKI, J. L.; GAIA, S. Gestão do conhecimento organizacional visando à transferência de tecnologia: os desafios enfrentados pelo NIT da Universidade Estadual de Santa Cruz. Produção Online, [S.l.], v. 13, n. 2, p. 77-702, 2013. 
SOUZA FILHO, H. M. et al. Condicionantes da Adoção de Inovações Tecnológicas na Agricultura.

Cadernos de Ciência e Tecnologia, Brasília, v. 28, n. 1, p. 223-255, jan.-abr., 2011.

VIEIRA FILHO. J. E. R. Difusão Biotecnológica: a adoção dos transgênicos na agricultura. Brasília: IPEA, 2014.

VITAL, T. W.; SILVA NETO, M. F. da. Transferência de tecnologias agropecuárias: fator de competitividade na microrregião de Petrolina, PE. Brasília: EMBRAPA, 2006.

\section{Sobre os Autores}

\section{Laíse Nunes Maciel}

E-mail: laisemaciel25@gmail.com

Engenheira de Produção. Graduada na Universidade Estadual de Santa Cruz (UESC).

Endereço profissional: Campus Soane Nazaré de Andrade, Rodovia Jorge Amado, km 16, Bairro Salobrinho, Ilhéus, BA. CEP: 45662-900.

\section{Lucas Xavier Trindade}

E-mail: lucas.xavier14@hotmail.com

Mestre em Economia Regional e Políticas Públicas (UESC) e Mestre em Conservação da Biodiversidade e Desenvolvimento Sustentável (IPÊ). Doutorando em Administração na Universidade de São Paulo (FEA/USP). Endereço profissional: Universidade de São Paulo (FEA/USP). Av. Prof. Luciano Gualberto, n. 908, Butantã, São Paulo, SP. CEP: 05508-010.

\section{Farlei Cosme Gomes dos Santos}

\section{E-mail: farlei.uesc@hotmail.com}

Mestre em Propriedade Intelectual e Transferência de Tecnologia para Inovação (PROFNIT/UESC). Professor do Centro Estadual de Educação Profissional do Chocolate Nelson Schaun.

Endereço profissional: Centro Estadual de Educação Profissional do Chocolate Nelson Schaun, Ilhéus, BA. CEP: 45650-015.

\section{João Pedro de Castro Nunes Pereira}

E-mail: jpcnpereira@uesc.br

Doutor em Engenharia de Produção (Poli/USP). Professor Pleno da Universidade Estadual de Santa Cruz - UESC. Endereço profissional: Campus Soane Nazaré de Andrade, Rodovia Jorge Amado, km 16, Bairro Salobrinho, Ilhéus, BA. CEP: 45662-900. 